\title{
Public knowledge and practice of sore throat management among visitors of primary care clinic in Riyadh, Saudi Arabia
}

\author{
Tamader Abdullatif Aloofy ${ }^{1 *}$, Lubna Al-Ansary ${ }^{2}$, Lama Ghassan Mokhlis ${ }^{3}$, Noha Khalid Khalil ${ }^{4}$, \\ Nouf Hatim Abo Alsamh ${ }^{5}$, Nourah Abdullatif Faden ${ }^{6}$, Sultana Mazen Borai ${ }^{7}$
}

1, 2, 3, 4, 5, 6, 7 King Saud University Hospital College of Medicine, Riyadh, Saudi Arabia

\section{Key Words:}

Sore throat management Pharyngitis

Acute respiratory tract infections

Primary care clinic

Received: 30 September 2016 Accepted: 10 October 2016

Published: 13 February 2017

\begin{abstract}
Sore throat is a common symptom of inflammation of the pharynx and tonsils, and it's a common health burden worldwide. The etiology in $85 \%$ of the time is a viral infection, which is a self-limiting disease. The other $15 \%$ of sore throat causes could be bacterial, fungal, smoking, intubation and yelling. Several studies were conducted regarding the management of pharyngitis and acute respiratory infections, which showed a high rate of antibiotic usage in an injudicious manner. This is a cross-sectional study, which took place in the primary care clinic in King Khalid University Hospital. A convenience sample was selected from the primary care clinic where data were collected via self-administered questionnaires. The research investigators designed the questionnaires and they were handed in 2014 in the time period between January 19 and January 27 where 320 questionnaires were handed. Data were collected from subjects who fit the inclusion criteria, which included adult outpatients (18 or above) who experienced sore throat in the past year. Details on the data that were collected include: demographic variables, clinical presentation, risk factors and mode of management. Data were analyzed using SPSS. The data collection procedure yielded 276 respondents; 44 participants were excluded for not being eligible or not fully filling the questionnaire. $52.2 \%$ of whom were females and $47.8 \%$ were males and only $6.9 \%$ of the total respondents chose the appropriate management which is analgesics. The age of respondents held no significance to the choice of management but gender exhibited significance as the majority of participants who chose the inappropriate management were males constituting $16.4 \%$ of the total. In addition, educational level as a demographic factor held significance as well in which respondents carrying bachelor's degrees formed a percentage of $14.3 \%$ of people who chose antibiotics while respondents carrying a high school degree chose analgesics establishing $2.9 \%$. The participants were asked if they follow any guidelines when managing their sore throat and $79.4 \%$ denied following guidelines. Also, we recommend similar research to be done on a bigger scale, with a bigger sample size and in different regions to know more about knowledge, attitude and practice of a bigger portion of the society. Moreover, further research is recommended regarding physicians and pharmacists prescribing antibiotics in an inappropriate fashion as well as supplying them with clear guidelines about the appropriate management of patients complaining of sore throat.
\end{abstract}

(C) 2017 The Author(s). Published by TAF Publishing.

\section{INTRODUCTION}

Sore throat is a common symptom of inflammation of the pharynx and tonsils, and it's a common health burden worldwide [1]. According to a study conducted in 1986 over a one-year period, 100 per 1000 people experienced sore throat a year [2]. The etiology in $85 \%$ of the time is a viral infection which is a self-limiting disease [3]. The other $15 \%$

\footnotetext{
${ }^{*}$ Corresponding author: Tamader Abdullatif Aloofy

†Email: tota_26@hotmail.com
} 
of sore throat causes could be bacterial, fungal, smoking, intubation and yelling [1]. The symptoms of sore throat may vary according to etiology; viral infections seem to be accompanied by a handful of symptoms including red swollen throat, trouble swallowing, swollen lymph nodes and fatigue. As for bacterial infections, it is believed that symptoms such as fever, swollen glands or lymph nodes and white spots or pus on the tonsils are usually associated [4]. Literature varied regarding sore throat management but the one recurrent controversial theme is that it is managed according to etiology; if sore throat is caused by a virus which is the cause in most cases, then it is self-limiting and the management shouldn't include antibiotics. If the cause is bacterial on the other hand, studies suggest that it's benign and self-limiting hence no antibiotic should be used. Other schools of thought suggest that antibiotics should be used to prevent complications. The British Medical Journal (BMJ) guidelines of sore throat management issued in 2012 showed that the appropriate and the most likely beneficial approach are analgesics such as Paracetamol [5]. National Health Service (NHS) guidelines supported this approach, along with some home remedies which may also relieve the symptoms [6]. Also, other guidelines suggested that no or delayed antibiotic prescribing strategy is recommended for patients experiencing acute sore throat among other conditions [7]. By the end of this study, we wish to determine the population's knowledge, attitude and practice regarding sore throat management in the hope of spreading awareness regarding inappropriate antibiotic use and the risk of serious complications induced by them amongst the Saudi population.

\section{Research Problem}

\section{Objectives and rationale of the study}

Our main objective was to assess the knowledge, atittude andpractice of a representative sample of Riyadh's population regarding sore throat management, thereby determining whether awareness efforts are needed regarding inappropriate antibiotic use and the risk of serious complications. The study specifically aimed to:

1. Determine the proportion of the population aware of the up-to-date evidence-based guidelines of sore throat management. By knowing this percentage, we either concur or discard the need for awareness of the proper management guidelines.

2. Describe the sore throat management practices of the visitors of primary care clinics in Riyadh, Saudi Arabia.

3. Identify the demographic factors (age, gender and educational level) that may affect the management choice; this helps in identifying and targeting the members of the population in need of awareness.

The results of this study will help in identifying areas to work on for correcting any misguided information through awareness on the proper guidelines of sore throat management, which will hopefully decrease the unnecessary complications of inappropriate management. Also, shed the light on the importance of sore throat management in future studies on a larger scale population, which will later on benefit the entire Saudi population.

\section{LITERATURE REVIEW}

We weren't able to obtain studies that focused on the people's knowledge about sore throat management and compared the difference in the people's management choice according to their demographic status. However, several studies were conducted regarding the management of pharyngitis and acute respiratory infections which showed a high rate of antibiotic usage in an injudicious manner. A study conducted in 2005 regarding the rate of antimicrobial drug usage in the ICU showed that $47.3 \%$ of the prescribed antibiotics were 
inappropriate and some were avoidable [8]. Another study showed that the rate of prescribed antibiotics to children with pharyngitis was very high in outpatient and emergency departments which led to the conduction of another study concerning sore throat management itself and the results showed that $53 \%$ of children were given antibiotics in excess to the expected prevalence of Group A Beta Hemolytic Streptococcus (GABHS). Some studies suggested that if the cause was GABHS after investigations then antibiotics should be prescribed to avoid complications but other studies counterpointed this theory by claiming that antibiotics do not reduce the risk of complications [9].

In a study conducted in 2005, the rate of antimicrobial use was measured and the numbers showed overuse of antimicrobials. However, the period from 1995 to 2003 showed that there was a decline in this overuse from $66 \%$ to $54 \%$. The decline is restricted to recommended antimicrobials i.e. in cases of GABHS, as for the non-recommended antimicrobials, they remain at constant $27 \%$ per visit which is considered high [10]. Furthermore, there were some studies that assessed the public view of antibiotic use not specifically in the context of sore throat management. One study done in 2006 in New Zealand showed that $38 \%$ are not aware that antibiotic use isn't helpful in vital infection [11]. In conclusion, there are some studies that describe the pattern of antibiotic prescription in sore throat and other studies that assess knowledge of antibiotic use in general. However, there are no studies that assess the general population's knowledge and practice of sore throat management.

\section{Research Question and Hypothesis Research question}

What is the public's level of knowledge and practice regarding sore throat management?

\section{Hypotheses}

-Null Hypothesis: Most of the population will abide by the guidelines in the management of sore throat with no significant demographic variation.

-Alternative hypothesis: We expect that most of the population does not follow proper guidelines in management of sore throat with respect to the demographic factors.

\section{METHODOLOGY}

This is a descriptive cross-sectional study, which took place in the time period from September 2013 to April 2014 at the Primary Care Clinics (PCCs) of King Khalid University Hospital (KKUH), Riyadh, Saudi Arabia. The primary care clinics treat both male and female patients above the age of 12 years old. It's a general clinic that is divided into three subclinics; refill, triage and follow up clinics and an extra clinic which is the gynecology clinic in the female side. The female primary care clinic receives around 130-140 patients each day and the male primary care clinic receives around 180 patients daily from around the country.

A pilot study was done on the 5th of November 2013 which served in determining the sample size and testing out the questionnaire's logic. The pilot questionnaires were handed to 5 female and 5 male patients/companions from the primary care clinic's waiting area. The feedback we got from the participants helped in editing and improving the questionnaire. Since we were unable to obtain any significant results about the people's knowledge and practice regarding sore throat management from previous literature to help in calculating the sample size, we used the appropriate formula to calculate the sample size according to the results of the pilot study which estimated the percentage of cor- 
rect management and a sample size of 246 was calculated accordingly. After putting a $25 \%$ no response and incomplete response rate into consideration, a sample size of 320 was estimated. Also, it holds room for the assessment of differences between men and women.

Our study's subjects were chosen according to convenience sampling technique in which data from subjects who fit the inclusion criteria were considered in the study results. This study included adult outpatients (18 or above) who are experiencing or have experienced sore throat within the year 2013. Children (because children don't usually choose their own treatment), in-patients, bedridden patients, patients with end stage diseases and those who never had an episode of sore throat in the past year were excluded from the study. Details on the data that were collected are:

Section one: Demographic variables which include age, occupation, educational level and gender. Section two: Clinical presentation and this includes fever, nasal congestion/runny nose, dry cough, muscle aches, redness of the eyes, enlarged tonsils, headache, rash, body ache/fatigue, sneezing, discomfort while swallowing, hoarseness of the voice, sputum and white patches inside the mouth. Section three: Risk factors including smoking, acid reflux, throat abuse, exposure to cold and hot weather and allergies. Section Four: Management and that consists of home remedies (herbs, cupping, honey, etc.), bed rest, over-the-counter medications (analgesics and antihistamines), a pharmacist's consultation and a physician's consultation.

Data were collected via self-administered questionnaires that the research investigators designed and modified according to the pilot study. The questionnaires were made in Arabic, the English translation was composed for research purposes. The investigators handed the questionnaires to visitors of the primary care clinic on the time period between January 19 and January 27 where 320 questionnaires were handed except on the days that coincided with the weekend because the clinic doesn't receive patients on weekends. In case of dealing with illiterate participants, the investigators assisted them in filling out the questionnaire on their behalf.

Data were analyzed using the SPSS program where all the variables (demography, symptoms, choice of management and the details associated) were inserted and the frequency tables of each variable generated. Cross tabulations between demographic variables and management choices were created and the chi square test for each cross tabulation was made to test if there are any significant differences in management options according to demographic variety. In terms of ethical consideration, the questionnaire included a cover page explaining the purpose of the study and the right of the participant to not reveal his/her identity. The participants' consent to use the information collected from them in our study was assumingly given by them agreeing to fill the questionnaire. Participants will remain anonymous and the information will be dealt with in confidentiality. There will be no incentives or rewards for people participating in the study. Also, there is no conflict of interest among the investigators of this study and the study was conducted as a part of the CMED 305 research course.

\section{RESULTS}

\section{Characteristics of Respondents}

The data collection procedure yielded 276 respondents; $52.2 \%$ of whom were females and $47.8 \%$ were males which means 44 people were excluded for either not being eligible or not fully filling the questionnaire. The age of participants ranged from 18 to 80 with a mean age of $37.04(+/-13.46)$. The respondents less than 25 years old showed the highest response rate with a percentage of $27.9 \%$ followed by the second highest rate of $27.2 \%$ 
which were people above the age of 46 years. As a whole, the highest proportion of the participants (41.8\%) were bachelor's degree graduates while less than $10 \%$ of the participants were illiterate.

As for occupational status, $43.6 \%$ were employees of different sectors excluding physicians who showed only $0.7 \%$ of responses. The rest of the participants were either unemployed or students of different specialties. Regarding chronic illnesses, the majority $(44.7 \%)$ reported having unspecified lung diseases followed by hypertension $(27 \%)$, while $26.2 \%$ reported having no chronic illnesses. Asthma, diabetes, high cholesterol and heart disease formed less than $50 \%$ of the cases. Moreover, most of the respondents were nonsmokers holding a very high rate of $84.8 \%$ (see figure 1 ).

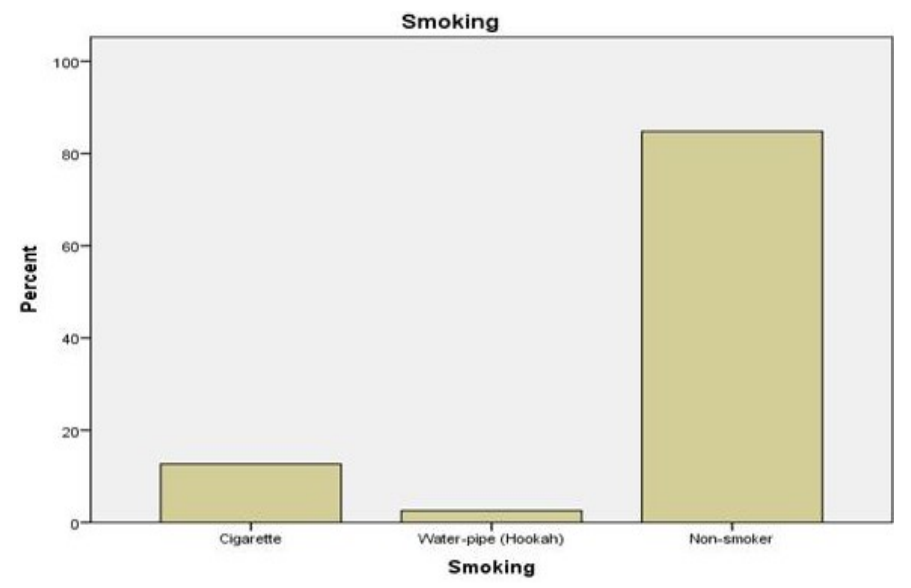

FIGURE 1. Smoking status of respondents

\section{Behavior of Respondents}

Most of the respondents (87.3\%) reported that they experienced sore throat less than 5 times a year but the majority (51.1\%) experienced it 2 times or less a year. Of all the people who experienced sore throat, more than half claimed that it usually lasts 1-4 days. When asked if the participants thought the cause of their sore throats was related to specific risk factors, the majority reported that smoking and acid reflux were never associated, whereas throat abuse (singing, shouting, coughing) and infections were sometimes related with flu and weather being more often so.

The symptoms experienced with the sore throat episode varied, the results reported by the majority are written below. They reported never experiencing white patches nor redness of the eye with their sore throats. On the other hand, fever was reported as sometimes experienced with the sore throat (39\%). However, the symptoms that were chosen to be as always associated with their sore throat are enlarged tonsils (33\%), body ache (34.1\%), headache (35.5\%), difficulty swallowing (45.7\%), hoarseness $(41.3 \%)$, runny or stuffy nose (41.9\%), cough (33.9\%) and sputum (36.5\%).

The choice of management varied between different types of modalities (see Table 1) including using analgesics only (6.9\%), using antibiotics whether alone or with other drugs (27.7\%), combination of bed rest with home remedies \& analgesics (16.8\%), visiting doctors \& pharmacists (0.7\%) and "others" which include antihistamine alone, bed rest alone and bed rest with home remedies (47.\%). Comparing the management choices between males and females, the results showed significant difference with a $\mathrm{p}$ value of 0.041 . More men preferred using antibiotics or using analgesics alone, with the figures being $16.4 \%$ and $4 \%$ respectively. However, more females (9.9\%) preferred using a com- 
TABLE 1. Doctors' and pharmacists' prescription

\begin{tabular}{|c|c|c|c|c|}
\hline \multicolumn{5}{|c|}{ Doctors'/Pharmacists' Management Frequencies } \\
\hline & & \multicolumn{2}{|c|}{ Responses } & \multirow[t]{2}{*}{ Percent of Cases } \\
\hline & & $\mathbf{N}$ & Percent & \\
\hline \multirow{10}{*}{$\begin{array}{l}\text { If you visited the doctor or } \\
\text { the pharmacist what did they prescribe? }\end{array}$} & Pharmacist prescription (Antibiotics) & 92 & $18.8 \%$ & $42.0 \%$ \\
\hline & Pharmacist prescription (Analgesics) & 9 & $1.8 \%$ & $4.1 \%$ \\
\hline & Pharmacist prescription (Vitamins) & 74 & $15.1 \%$ & $33.8 \%$ \\
\hline & Pharmacist prescription (Antihistamines) & 17 & $3.5 \%$ & $7.8 \%$ \\
\hline & Pharmacist prescription (No Medication) & 5 & $1.0 \%$ & $2.3 \%$ \\
\hline & Doctor's prescription (Antibiotics) & 156 & $31.8 \%$ & $71.2 \%$ \\
\hline & Doctor's prescription (Analgesics) & 9 & $1.8 \%$ & $4.1 \%$ \\
\hline & Doctor's prescription (Vitamins) & 93 & $19.0 \%$ & $42.5 \%$ \\
\hline & Doctor's prescription (Antihistamines) & 33 & $6.7 \%$ & $15.1 \%$ \\
\hline & Doctor's prescription (No Medication) & 2 & $0.4 \%$ & $0.9 \%$ \\
\hline Total & & 490 & $100 . \%$ & $223.7 \%$ \\
\hline
\end{tabular}

bination of analgesics, bed rest and home remedies. The females also outnumbered the males who chose "others" as a choice of management (28.1\%) for females versus $(19.7 \%)$ for males (See figure 2).



FIGURE 2. Choice of management according to gender

As for comparison of management choice in different age groups, the age showed no significance with a $\mathrm{p}$ value of 0.865 . On the other hand, educational level was significant ( $p$ value $=0.039)$. The majority of high school graduates $(2.9 \%)$ chose analgesics while the majority of people who held bachelor's degree (14.3\%) chose antibiotics. Most of the illiterate participants (2.9\%) chose "others". When asked whether the subject visits the doctor each time they experience sore throat, the majority replied no with a percentage of $78 \%$.

On the subject of visiting the doctor or the pharmacist, only $1.8 \%$ of both prescribed analgesics, while $31.8 \%$ of doctors and $18.8 \%$ of pharmacists prescribed antibiotics (see table 1). Among the doctors, who prescribed antibiotics, only $3.3 \%$ ordered a throat bacterial culture.

Out of the people who took over-the-counter medication, 58.4\% reported that they were influenced by previous experience from a visit to the doctor. To conclude, the participants were asked if they follow any guidelines when managing their sore throat and $79.4 \%$ denied following guidelines. 


\section{DISCUSSION AND LIMITATIONS \\ Discussion}

In our study, we measured the knowledge, attitude and practice of people visiting the primary care clinic in KKUH regarding sore throat management and thus determining the people's consistency with the guidelines and their need for awareness. We received a response rate of $86 \%$ which is considered good, but shockingly only $6.9 \%$ of respondents chose the appropriate management based on the previously mentioned guidelines, which are analgesics [7]. However, some people chose more than one approach that included bed rest, home remedies and analgesics, which accounted for $16.8 \%$ and this may categorize them on a somewhat correct level of knowledge, based on the recommendations of other guidelines [8] i.e. state analgesics and self-care as an appropriate management entity (see table 2). Based on the previous literature [7-8] the majority showed an agreement

TABLE 2. Choice of management

\begin{tabular}{llcccc}
\hline \hline \multicolumn{7}{c}{ When experiencing these symptoms do you prefer } & & \\
\hline & & Frequency & Percent & Valid Percent & Cumulative Percent \\
\hline \multirow{4}{*}{ Valid } & Using analgesics only & 19 & 6.9 & 6.9 & 6.9 \\
& Using antibiotics only, using Antibiotics \& analgesics, Antibiotics \& antihistamines & 76 & 27.5 & 27.7 & 34.7 \\
& Bed rest \& home remedies \& analgesics & 46 & 16.7 & 16.8 & 51.5 \\
& Visiting Doctors \& pharmacists & 2 & .7 & .7 & 52.2 \\
\hline Total & Others & 131 & 47.5 & 47.8 & 100.0 \\
\hline \hline
\end{tabular}

in that the use of antibiotics is not recommended and some would say controversial. This is relevant to our study because $27.7 \%$ of our sample chose antibiotics as a method of management opposing the guidelines. Even though not all of the remaining $72.3 \%$ chose the correct management (analgesics), the fact that they didn't choose antibiotics is considered somewhat good practice. In this study, one of the objectives focused on demographic factors and their relation to the choice of management. One of our demographic factors to be discussed is gender, which showed significance. The greater number of participants who chose the appropriate management which is analgesics were males, constituting $4.0 \%$ but they also were the majority that chose antibiotics with a high percentage of $16.4 \%$ which is worth highlighting because according to evidence base [8], it's not compatible. However, out of the total percentage, $16.8 \%$ of people chose analgesics along with bed rest and home remedies, $9.9 \%$ were female which is again considered good practice according to NHS guidelines [7].

Lastly, educational level as a demographic factor holds significance, which means it could be a possible predictor for management choice pattern. Surprisingly, respondents carrying a high school degree were the people who chose the correct approach i.e. analgesics constituting $2.9 \%$. The people who chose analgesics as well as bed rest and home remedies at a percent of $6.6 \%$ are the ones with bachelor's degrees making them adherent to some guidelines, but they also formed a percentage of $14.3 \%$ of participants who chose antibiotics as first line management which is considered unusual. Although previous literature showed physician prescribing antibiotics in excess $[9,11]$ and our results showed that in most cases where people visited the doctor they were prescribed antibiotics, only $27.7 \%$ of our respondents were taking antibiotics which may show some disregard to doctor's prescriptions. Fortunately, in this case the people's disregard to doctor's prescriptions is good for their well-being. In conclusion, based on our results, we reject the null hypothesis therefore accepting the alternative hypothesis, thus confirming their need for awareness due to the lack of knowledge and adherence to proper guidelines. As for the sub objective concerning the effect of demographic factors on the choice of man- 
agement, gender and educational level held significant difference upon choosing management, unlike age which bared no significance.

\section{Limitations}

The research had several potential limitations but it was primarily related to the sample. We followed a convenience sampling technique not representative of the whole population but only limited to those visiting KKUH primary care clinic. Also, extra questionnaires were handed increasing our sample size from 246 to 320 in cases of loss of questionnaire or incomplete responses. Lastly, the distributing of questionnaires was held in nonconsecutive days due to the fact that the primary care clinics do not receive patients on weekends.

\section{CONCLUSION AND RECOMMENDATIONS}

Efforts to increase awareness regarding sore throat management are still needed in general but should be particularly targeted towards males and those who hold bachelor's degree since they showed high percentages of the wrong management choice (antibiotics). Also, we recommend similar research to be done on a bigger scale, with a bigger sample size and in different regions to know more about knowledge, attitude and practice of a bigger portion of the society. Moreover, further research is recommended regarding doctors and pharmacists prescribing antibiotics in an inappropriate fashion as well as supplying them with clear guidelines about the appropriate management of patients complaining of sore throat. Hopefully, these recommendations will help our society flourish.

\section{Acknowledgment}

First, we thank our supervisor Professor Lubna Al-Ansary for her guidance. We would also like to thank Ruah Al-Yamany for sharing her knowledge and her assistance in every step of this research. Moreover, we would like to thank Dr. Shikh Shafi for his help in the statistical calculations and Saleh Mohammed Abu Al-gaith for his help in accessing guidelines and papers that were essential in our research. Also, we would like to thank our families for their ongoing support. Finally, we would like to thank all the people who participated by filling the questionnaires.

\section{REFERENCES}

1. Lynm C, Parmet S, Glass R. Sore throat. JAMA. 2004; 291(13): 16-64.

2. Hoffmann S. Incidence and management of sore throat in general practice. Scandinavian Journal of Primary Healthcare. 1986; 4(3): 143-150. DOI: 10.3109/02813438609014821

3. Worrall GJ. Acute sore throat. Canadian Family Physician. 2007; 53(11): 19-61.

4. NPS Medicinewise. How is a throat infection diagnosed? n.d. Available from: goo.gl/GZ4uic

5. Kenealy T. Sore throat. British Medical Journal. 2007; 11(1509): 1-10.

7. NHS. Sore throat treatment national health service. 2012. Available from: goo.gl/AC41CY

8. Erbay A, Bodur H, Akıncı E, Colpan A. Evaluation of antibiotic use in intensive care units of a tertiary care hospital inTurkey. Journal of Hospital Infection. 2005; 59(1): 53-61. DOI: 10.1016/j.jhin.2004.07.026

9. Regoli M, Chiappini E, Bonsignori F, Galli L, de Martino M. Update on the management of acute pharyngitis in children. Italian Journal of Pediatrics. 2011; 37(1): 1-10. DOI: 10.1186/1824-7288-37-10

10. Weber JT. Appropriate use of antimicrobial drugs: A better prescription is needed. JAMA. 2005; 294(18): 2354-2356. DOI: $10.1001 /$ jama.294.18.2354

11. Curry M, Sung L, Arroll B, Goodyear-Smith F, Kerse N, Norris P. Public views and use of antibiotics for the common cold before and after an education campaign in New Zealand. The New Zealand Medical Journal. 2006; 5(119): 1233. 